\title{
Systematics of the electric dipole response in stable tin isotopes
}

\author{
Sergej Bassauer ${ }^{1, \star}$, Peter von Neumann-Cosel ${ }^{1}$, and Atsushi Tamii ${ }^{2}$ \\ ${ }^{1}$ Institut für Kernphysik, Technische Universität Darmstadt, 64289 Darmstadt, Germany \\ ${ }^{2}$ Research Center for Nuclear Physics, Osaka University, Ibaraki, Osaka 567-0047, Japan
}

\begin{abstract}
The electric dipole is an important property of heavy nuclei. Precise information on the electric dipole response provides information on the electric dipole polarisability which in turn allows to extract important constraints on neutron-skin thickness in heavy nuclei and parameters of the symmetry energy. The tin isotope chain is particularly suited for a systematic study of the dependence of the electric dipole response on neutron excess as it provides a wide mass range of accessible isotopes with little change of the underlying structure. Recently an inelastic proton scattering experiment under forward angles including $0^{\circ}$ on ${ }^{112,116,124} \mathrm{Sn}$ was performed at the Research Centre for Nuclear Physics (RCNP), Japan with a focus on the low-energy dipole strength and the polarisability. First results are presented here. Using data from an earlier proton scattering experiment on ${ }^{120} \mathrm{Sn}$ the gamma strength function and level density are determined for this nucleus.
\end{abstract}

\section{Introduction}

The electric dipole response in heavy nuclei is dominated by the giant dipole resonance (GDR), a collective mode above the particle emission threshold usually described as an oscillation of protons against neutrons. Around the neutron threshold the electric dipole response shows itself as an oscillation of a neutron skin against an isospin saturated core. This mode is called pigmy dipole resonance (PDR). The GDR can be investigated using $(\gamma, \mathrm{xn})$ experiments, whereas the investigation of the PDR is more complicated. Due to its occurrence around the neutron threshold, it can be accessed by $\left(\gamma, \gamma^{\prime}\right)$ experiments below the neutron threshold and by $(\gamma, \mathrm{xn})$ experiments above the neutron threshold. However, inelastic proton scattering experiments under $0^{\circ}$ allow to investigate the GDR and PDR in a single experiment.

The electric dipole response provides information on the electric dipole polarisability which in turn allows to extract important constraints on the neutron-skin thickness [1-5] in heavy nuclei and parameters of the symmetry energy [2, 6-8]. Another important property of nuclei which can be extracted from the electric dipole response is the gamma strength function (GSF). GSFs describe the average gamma decay behaviour of a nucleus as a function of gamma energy. They serve as input in calculations of cross sections in astrophysics [9], reactor design [10] and waste transmutation [11] within statistical models. Using the so called fluctuation analysis [12] it is also possible to determine level densities in the GDR region [13].

\footnotetext{
^e-mail: sbassauer@ikp.tu-darmstadt.de
}
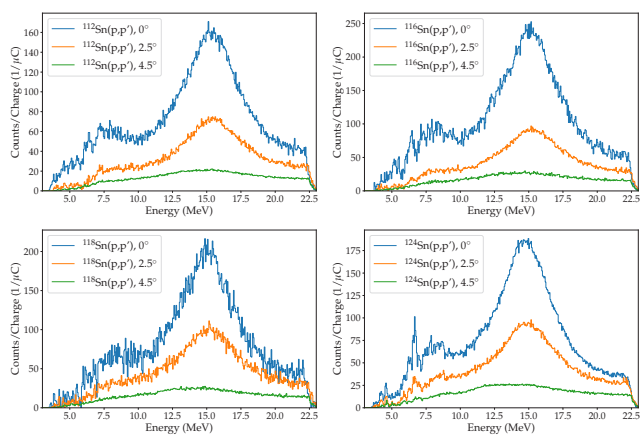

Figure 1. (Colour online) Inelastic proton scattering spectra under laboratory scattering angles of $0^{\circ}, 2.5^{\circ}$ and $4.5^{\circ}$ normalised on the collected charge for ${ }^{112,116,118,124} \mathrm{Sn}$.

\section{Experiment and preliminary results}

The proton scattering experiment was performed at the Research Centre for Nuclear Physics (RCNP), Japan. In this experiment unpolorised protons with a beam energy of $295 \mathrm{MeV}$ and a beam current of up to $6 \mathrm{nA}$ were used. An energy resolution of about $40 \mathrm{keV}$ could be achieved. Data was taken at laboratory scattering angles of $0^{\circ}, 2.5^{\circ}$ and $4.5^{\circ}$. The main targets were ${ }^{112} \mathrm{Sn},{ }^{116} \mathrm{Sn}$ and ${ }^{124} \mathrm{Sn}$. Additionally some data on ${ }^{118} \mathrm{Sn}$ could be taken. Details on the experimental set-up and data analysis can be found in [14].

In Fig. 1, inelastic proton scattering data for $0^{\circ}$, $2.5^{\circ}$ and $4.5^{\circ}$ normalised on the collected charge for $112,116,118,124 \mathrm{Sn}$ can be seen. With increasing angle the typical decrease in the cross section due to the dominant 


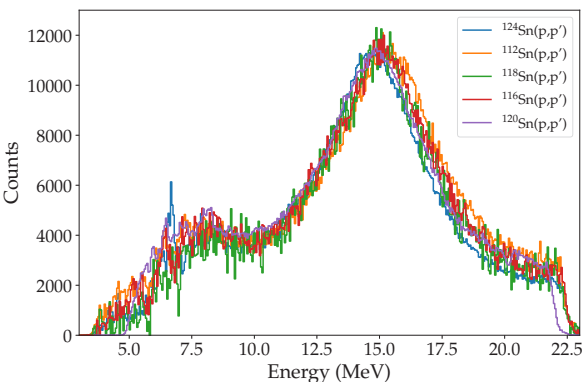

Figure 2. (Colour online) Comparison of the ${ }^{112,116,118,120,124} \mathrm{Sn}$ spectra (normalised on ${ }^{124} \mathrm{Sn}$ ). The data for ${ }^{120} \mathrm{Sn}$ stems from $[15,16]$.

Coulomb interaction under small angles can be observed. In all isotopes the GDR can be clearly identified around $15 \mathrm{MeV}$. Around the neutron threshold a bump can be seen. In ${ }^{124} \mathrm{Sn}$ strong structure is apparent in the low energy region.

Fig. 2 shows a comparison between the measured isotopes and ${ }^{120} \mathrm{Sn}$ from an earlier experiment [15]. For comparison all data was normalised on ${ }^{124} \mathrm{Sn}$. As expected the centroid energy of the GDR is the lowest for ${ }^{124} \mathrm{Sn}$. It is shifted to higher energies for lighter tin isotopes and is highest for ${ }^{112} \mathrm{Sn}$. All spectra show a bump around $8 \mathrm{MeV}$ and a structure around $6.5 \mathrm{MeV}$, most pronounced for ${ }^{124} \mathrm{Sn}$

\section{Gamma strength function and level density of ${ }^{120} \mathrm{Sn}$}

The double differential cross section for ${ }^{120} \mathrm{Sn}$ is available from an earlier proton scattering experiment $[15,16]$. Using the so called virtual photon method the double differential cross section can be converted to photoabsorption cross section which allows to determine the E1 part of the GSF [17]. The M1 part of the GSF was extracted from M1 cross sections [15]. In Fig. 3 the total gamma strength function for ${ }^{120} \mathrm{Sn}$ is shown. In the GDR region the GSF from proton scattering is compared to GSFs from $(\gamma, \mathrm{xn})$ experiments [19-21]. The agreement in this region is very good. In the low energy region gamma strength functions from $\left({ }^{3} \mathrm{He},{ }^{3} \mathrm{He}^{\prime} \gamma\right)$ and $\left({ }^{3} \mathrm{He}, \alpha \gamma\right)$ for ${ }^{116,118,122} \mathrm{Sn}$ are shown $[22,23]$. Unfortunately no GSF for ${ }^{120} \mathrm{Sn}$ from this type of experiments is available. In comparison to ${ }^{120} \mathrm{Sn}$ from proton scattering the agreement is quite well in the low energy region. However, two bumps can be seen in the GSF from proton scattering around $6.5 \mathrm{MeV}$ and $8.3 \mathrm{MeV}$. Around 8.3 MeV no direct comparison can be made since experimental data from gamma decay experiments are limited to lower energies. The bump around $6.5 \mathrm{MeV}$ seems to be enhanced compared to $\left({ }^{3} \mathrm{He}^{3}{ }^{3} \mathrm{He}^{\prime} \gamma\right)$ and $\left({ }^{3} \mathrm{He}, \alpha \gamma\right)$ experiments (note the logarithmic scale of Fig. 3 ). This could be an indication of a violation of the Brink-Axel hypothesis (in contrast to a recent analysis in ${ }^{96} \mathrm{Mo}$ with the same

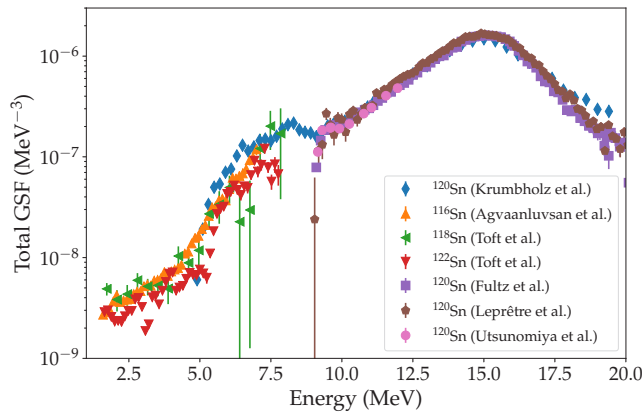

Figure 3. (Colour online) Total gamma strength function of ${ }^{120} \mathrm{Sn}$ in comparison to gamma strength functions from $(\gamma, \mathrm{xn})$, $\left({ }^{3} \mathrm{He},{ }^{3} \mathrm{He}^{\prime} \gamma\right)$ and $\left({ }^{3} \mathrm{He}, \alpha \gamma\right)$ experiments [19-23].

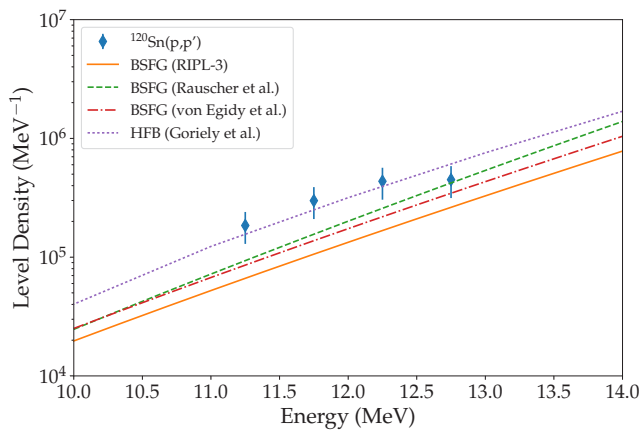

Figure 4. (Colour online) Level density of ${ }^{120} \mathrm{Sn}$ for $1^{-}$states.

technique [24]), which states that the GSF depends only on the gamma energy and is independent of the structure of the initial and final state $[25,26]$.

Using a fluctuation analysis [12] it was possible to extract level densities in the GDR region for $1^{-}$states as shown in Fig 4. The results are compared to Backshifted Fermi Gas Model (BSFG) calculations with different parametrisations and a Hartree-Fock-Bogoliubov Model (HBF) results [27-30]. From $\left({ }^{3} \mathrm{He}^{3}{ }^{3} \mathrm{He}^{\prime} \gamma\right)$ and $\left({ }^{3} \mathrm{He}, \alpha \gamma\right)$ experiments only total level densities can be extracted. Therefore the level densities from proton scattering for $1^{-}$states must be converted to total level densities for a comparison. The procedure for this conversion is described in [31]. In Fig. 5 the total level density in ${ }^{120} \mathrm{Sn}$ from proton scattering is compared to the level densities from $\left({ }^{3} \mathrm{He}^{3}{ }^{3} \mathrm{He}^{\prime} \gamma\right)$ and $\left({ }^{3} \mathrm{He}, \alpha \gamma\right)$ experiments in neighbouring tin isotopes. In the low energy region the BSFG models reproduce the level densities quite well, whereas at higher energies the HFB model seems to describe the level densities more accurately. 


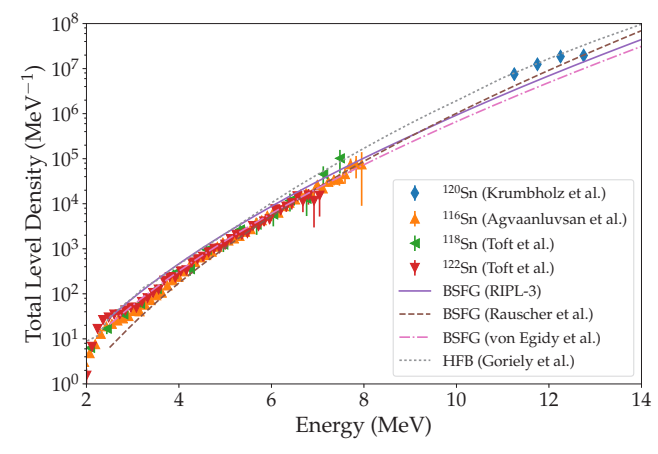

Figure 5. (Colour online) Total level density from proton scattering compared to the level densities from $\left({ }^{3} \mathrm{He}^{3}{ }^{3} \mathrm{He}^{\prime} \gamma\right)$ and $\left({ }^{3} \mathrm{He}, \alpha \gamma\right)$ experiments.

\section{Summary and conclusions}

First results from a recent inelastic proton scattering experiment on ${ }^{112,116,118,124} \mathrm{Sn}$ are presented. In all isotopes the GDR can be clearly identified. Around the neutron threshold a bump can be seen. Particularly in ${ }^{124} \mathrm{Sn}$ strong structure is visible at about $6.5 \mathrm{MeV}$. The expected shift of the centroid energy of the GDR can be observed in the tin isotope chain.

The gamma strength function and level density for ${ }^{120} \mathrm{Sn}$ was determined from the data of Refs. $[15,16]$. The GSF agrees well in the GDR region with other experiments. In the low energy region an increase around $6.5 \mathrm{MeV}$ can be seen in the GSF from proton scattering. This is in disagreement with the GSFs from $\left({ }^{3} \mathrm{He},{ }^{3} \mathrm{He}^{\prime} \gamma\right)$ and $\left({ }^{3} \mathrm{He}, \alpha \gamma\right)$ experiments (albeit not for ${ }^{120} \mathrm{Sn}$ but for neighbouring tin isotopes), which could be an indication of a Brink-Axel hypothesis violation.

The level densities agree well with the HFB model at higher energies. At lower energies however, a better agreement with the BSFG model is observed.

\section{Acknowledgements}

We thank all collaborators of the E316 and E422 experiments. This work was supported by the DFG under con- tract SFB 1245 and by the JSPS, KAKENHI Grant No. JP25105509.

\section{References}

[1] J. Piekarewicz, Phys. Rev. C 73, 044325 (2006).

[2] A. Klimkiewicz et al., Phys. Rev. C 76, 051603(R) (2007).

[3] N. Tsoneva et al., Phys. Rev. C 77, 024321 (2008).

[4] J. Piekarewicz, Phys. Rev. C 83, 034319 (2011).

[5] T. Inakura et al., Phys. Rev. C 84, 021302(R) (2011).

[6] A. Carbone et al., Phys. Rev. C 81, 041301(R) (2010).

[7] F.J. Fattoyev et al., Phys. Rev. C 86, 015802 (2012).

[8] M. B. Tsang et al., Phys. Rev. C 86, 015803 (2012).

[9] M. Arnould et al., Phys. Rep. 450, 97 (2007).

[10] M.B. Chadwick et al., Nucl. Data Sheets 112, 2887 (2011).

[11] M. Salvatore et al., Prog. Part. Nucl. Phys. 66, 144 (2011).

[12] P.G. Hansen et al., Nucl. Phys. A 518, 13 (1990).

[13] I. Poltoratska et al., Phys. Rev. C 89, 054322 (2014).

[14] A. Tamii et al., Nucl. Instrum. Methods Phys. Res., Sect. A 605, 3 (2009).

[15] A.M. Krumbholz, et al., Phys. Lett. B 744, 7 (2015).

[16] T. Hashimoto et al., Phys. Rev. C 92, 031305 (2015).

[17] C. Bertulani et al., Phys. Rep. 163, 299 (1988).

[18] J. Birkhan et al., Phys. Rev. C 93, 041302(R) (2016).

[19] A. Leprêtre et al., Nucl. Phys. A 219, 39 (1974).

[20] S.C. Fultz et al., Phys. Rev. 186, 1255 (1969).

[21] H. Utsunomiya et al., Phys. Rev. C 84, 055805 (2011).

[22] U. Agvaanluvsan et al., Phys. Rev. C 79, 014320 (2009).

[23] H.K. Toft et al., Phys. Rev. C 83, 044320 (2011).

[24] D. Martin et al., Phys. Rev. Lett. 119, 182503 (2017).

[25] D.M. Brink, Ph.D. thesis, Oxford University (1955).

[26] P. Axel, Phys. Rev. 126, 671 (1962).

[27] R. Capote et al., Nucl. Data Sheets 110, 3107 (2009).

[28] T. Rauscher, Phys. Rev. C 78, 032801 (2008).

[29] T. von Egidy et al., Phys. Rev. C 80, 054310 (2009).

[30] S. Goriely, et al., Phys. Rev. C 78, 064307 (2008).

[31] S. Bassauer, et al., Phys. Rev. C 94, 054313 (2016). 